\title{
BMJ Open Protocol for surgical and non-surgical treatment for metacarpal shaft fractures in adults: an observational feasibility study
}

Rowa Taha (D) , ${ }^{1}$ Paul Leighton, ${ }^{2}$ Chris Bainbridge, ${ }^{3}$ Alan Montgomery, ${ }^{4}$ Tim Davis, ${ }^{5}$ Alexia Karantana ${ }^{6}$

To cite: Taha R, Leighton $P$, Bainbridge $\mathrm{C}$, et al. Protocol for surgical and nonsurgical treatment for metacarpal shaft fractures in adults: an observational feasibility study. BMJ Open 2021;11:e046913. doi:10.1136/ bmjopen-2020-046913

- Prepublication history for this paper is available online. To view these files, please visit the journal online (http://dx.doi. org/10.1136/bmjopen-2020046913).

Received 12 November 2020 Accepted 15 April 2021

Check for updates

(C) Author(s) (or their employer(s)) 2021. Re-use permitted under CC BY. Published by BMJ.

${ }^{1}$ Academic Orthopaedics, Trauma \& Sports Medicine, University of Nottingham School of Medical and Surgical Sciences, Nottingham, UK

${ }^{2}$ Division of Primary Care, University of Nottingham, Nottingham, UK

${ }^{3}$ Pulvertaft Hand Centre, Royal Derby Hospital, Derby, UK ${ }^{4}$ Nottingham Clinical Trials Unit, University of Nottingham,

Nottingham, UK

${ }^{5}$ Trauma and Orthopaedics, Nottingham University Hospitals NHS Trust, Nottingham, UK

${ }^{6}$ Surgery, University of

Nottingham Faculty of

Medicine and Health Sciences, Nottingham, UK

Correspondence to

Rowa Taha;

rowa.taha@nottingham.ac.uk

\section{ABSTRACT}

Introduction Metacarpal shaft fractures (MSF) are common traumatic hand injuries that usually affect young people of working age. They place a significant burden on healthcare resources and society; however, there is a lack of evidence to guide their treatment. Identifying the most beneficial and cost-efficient treatment will ensure optimisation of care and provide economic value for the National Health Service. The aim of this study is to assess the feasibility of a randomised controlled trial comparing surgical and non-surgical treatment for MSF in adults.

Methods and analysis This is a multicentre prospective cohort study, with a nested qualitative study consisting of patient interviews and focus groups, and an embedded factorial randomised substudy evaluating the use of text messages to maximise data collection and participant retention. The outcomes of interest include eligibility, recruitment and retention rates, completion of follow-up, evaluation of primary outcome measures, calculation of the minimal clinically important difference (MCID) for selected outcome measures and establishing the feasibility of data collection methods and appropriate time-points for use in a future trial. Data will be captured using a secure online data management system. Data analyses will be descriptive and a thematic inductive analysis will be used for qualitative data. Minimum clinically important effects for each patient-reported outcome measure will be estimated using anchor-based responsiveness statistics and distribution-based methods.

Ethics and dissemination This study has received ethical approval from the Research Ethics Committee and the Health Research Authority (REC reference 20/EE/0124). Results will be made available to patients, clinicians, researchers and the funder via peer-reviewed publications and conference presentations. Social media platforms, local media and feedback from the Patient Advisory Group will be used to maximise circulation of findings to patients and the public.

Trial registration number ISRCTN13922779.

\section{INTRODUCTION}

Metacarpal shaft fractures (MSF) are common traumatic hand injuries reported to represent $18 \%-31 \%$ of hand fractures. ${ }^{1-4}$ They usually affect young adult males, often in the third
Strengths and limitations of this study

- Provides comprehensive information to inform the design and implementation of a future trial comparing treatments for metacarpal shaft fractures (MSF), including evaluation of multiple outcome measures and calculation of minimal clinically important difference to inform future sample size calculations.

- Provides complementary person-centred insight into MSF and research design, conduct and delivery through embedded qualitative assessments.

- Evaluates effective text message strategies for maximising data collection and retention in research studies.

- A detailed cost evaluation is a particular strength of the study and will deliver a comprehensive analysis of the costs of treatments available for MSF.

- Limited assessment of randomisation.

decade of life, ${ }^{15}$ with the fourth and fifth metacarpal most commonly injured. ${ }^{1-6}$

In 2016, there were 23.5 million accident and emergency (A\&E) department attendances in the UK with fractures being the second most common reason for presentation. ${ }^{7}$ As hand fractures make up $25 \%$ of all A\&E attendances ${ }^{8}$ and MSF comprise $18 \%-31 \%$ of hand fractures, ${ }^{1-4}$ MSF therefore place a significant burden on healthcare resources.

MSF predominantly affect those of working age $^{2-489}$ and are thus associated with significant cumulative morbidity. ${ }^{10}$ Missed time off work significantly increases the economic burden of $\mathrm{MSF}^{10}$ However, there is no UK-specific data on the healthcare-associated costs, socioeconomic, or societal costs of these injuries.

There is wide variability in the management of MSF. MSF can be managed non-surgically with appropriate reduction and immobilisation, ${ }^{11} 12$ or surgically using a variety of 
different techniques, including Kirschner-wires (K-wires), intraosseous wires, interfragmentary compression screws, plates or external fixators. ${ }^{13}$

A systematic review undertaken in 2019 of treatment interventions for MSF identified 699 records and no randomised controlled trials (RCTs) comparing surgical to non-surgical treatments. ${ }^{14}$ The only retrospective cohort study had several key limitations including small patient numbers, low follow-up rate $(17 \%)$ and lack of use of a patient-reported outcome measure (PROM) validated in MSF. ${ }^{15}$ A search of the WHO ISCTRP portal also revealed no ongoing or registered trials worldwide. ${ }^{14}$

\section{Rationale for study}

There is a lack of good quality, large comparative trials to guide the treatment of MSF. There are no published or ongoing RCTs or cohort studies comparing surgical versus non-surgical treatment for MSF. In addition, though the use of PROMs in both the clinical and research setting has increased in recent years, there is no evidence of reliability, validity and responsiveness of PROMs in MSF.

There are several gaps in the literature:

- No consensus on acceptable parameters of deformity or displacement, leading to widespread variation in treatment

- No core outcome sets for hand trauma, so we do not know which outcome measures are best suited for the study of MSF

- No qualitative data exploring patient experience of MSF and their treatment

- No evaluation of the cost-effectiveness of treatment modalities for MSF

- No high-quality published evidence comparing treatment modalities for MSF

The lack of existing evidence supports the need for a well-designed, pragmatic, multicentre RCT to identify the most beneficial and cost-efficient treatment for MSF in adults. This study aims to assess the feasibility, acceptability and practicality of such a trial by providing information about study design, number of eligible patients, recruitment, completion of follow-up, selection of appropriate outcome measures, assessment of minimal clinically important difference (MCID) for selected outcome measures, costs of treatments and measures to optimise recruitment, engagement and retention in a future trial.

\section{Study objectives and purpose}

The overall purpose of this study is:

1. To investigate the feasibility and acceptability of conducting a multicentre RCT to assess the clinical and cost-effectiveness of surgical and non-surgical treatment for MSF in adults.

2. To provide complementary, detailed and personcentred insight that will inform RCT design through the identification of barriers to participation among patients with MSF, and to develop novel solutions to engage these cohorts in research.
The objectives of the study (table 1) were developed at a MSF Consensus Workshop, held by the Centre for Evidence Based Hand Surgery in Nottingham, November 2018 involving patients, clinicians, therapists and clinical trials methodologists. ${ }^{16}$

\section{METHODS AND ANALYSIS}

\section{Study configuration}

FACTS is an observational, multicentre, prospective cohort study. Treatments will not be randomly allocated; patient care will be determined in the usual way as per the treating clinician.

Patients who meet the eligibility criteria (table 2) will be recruited from hand fracture clinics at participating National Health Service (NHS) hospitals. Where participants have multiple MSF with potentially variable fracture patterns, a single digit will be selected as the 'study finger'. Participants with additional hand or upper limb injuries will be included and the latter recorded. Written informed consent will be obtained from all participants by an appropriately trained research associate prior to entering the study. Thereafter, participants will be reviewed in a face-to-face clinic visit at 6 weeks and remotely at 3 and 6 months. This was supported by previous studies of MSF reporting full range of motion in almost all patients by 6 months. ${ }^{17}$ Furthermore, previous studies in similar patient cohorts with follow-up of 12 months or longer suffered high drop-rates or struggled to recruit adequate numbers of participants. A participant pathway flowchart is illustrated in figure 1 .

A nested qualitative study consisting of two elements, patient interviews and focus groups, will be conducted to provide patient-centred insight into study procedures and explore the individual impact of the injury. Participants will be selected from the prospective cohort study, using purposive sampling that prioritises young males, and further written informed consent separately sought for this element of the study.

A detailed cost evaluation to establish the costs of treatments for MSF through representative micro-costing will be undertaken. Resource use directly linked to the MSF and its sequela and/or complications over the 6 months of follow-up will be recorded for each participant. Unit cost data will be obtained from national databases such as NHS reference costs, the British National Formulary (BNF) and Personal Social Services Research Unit (PSSRU) Costs of Health and Social Care. ${ }^{18}$

A two by two by two factorial randomised substudy will be nested within the main cohort study. Once participants have consented to the cohort study or qualitative study, they will be randomised to the following interventions to evaluate the use of text messages in maximising data collection and participant retention; frequency of text messages-either fortnightly or monthly; two-way communication-text message requiring a response from the participant versus a notification message only; and personalisation-a personalised message versus a standard automated message. 


\section{Table 1 Study objectives}

\begin{tabular}{|c|c|}
\hline How objective will inform the definitive trial & Objective \\
\hline Recruitment for a future trial & $\begin{array}{l}\text { 1. Define eligibility criteria for the future trial, which correctly identify appropriate } \\
\text { patients for whom a treatment decision is suitable } \\
\text { 2. Estimate the proportion of referred NHS patients who meet these eligibility } \\
\text { criteria } \\
\text { 3. Assess recruitment and retention rates }\end{array}$ \\
\hline Outcomes for use in a future trial & $\begin{array}{l}\text { 4. Evaluate outcomes for use as primary and secondary outcomes } \\
\text { 5. Calculate minimal clinically important difference for the proposed primary } \\
\text { outcome measure } \\
\text { 6. Investigate feasibility of collecting outcome data frequently, in order to capture } \\
\text { subtle improvements in patient-assessed or clinician-assessed outcomes }\end{array}$ \\
\hline Follow-up & $\begin{array}{l}\text { 7. Estimate follow-up and outcome completion rates for both clinic and remotely } \\
\text { assessed outcomes } \\
\text { 8. Explore optimum time-points for follow-up }\end{array}$ \\
\hline Sample size calculation & 9. Estimate the sample size required for a definitive study \\
\hline Economic assessment & $\begin{array}{l}\text { 12. Inform the design of a future cost-effectiveness analysis by exploring the } \\
\text { costs of treatment modalities through capture of NHS resource use and } \\
\text { representative micro-costing }\end{array}$ \\
\hline $\begin{array}{l}\text { Patient-centred insight into research design, } \\
\text { conduct and delivery }\end{array}$ & $\begin{array}{l}\text { 13. To explore participant experience of MSF, treatment and recovery } \\
\text { 14. To explore participant experience of research processes and study burden } \\
\text { associated with outcome measures } \\
\text { 15. To gain recommendations on future study design and mechanisms to facilitate } \\
\text { study delivery }\end{array}$ \\
\hline $\begin{array}{l}\text { Facilitate engagement and retention among } \\
\text { patients with MSF in a future trial }\end{array}$ & $\begin{array}{l}\text { 16. To explore the use of health technology applications and social media in } \\
\text { optimising participation and engagement in research }\end{array}$ \\
\hline
\end{tabular}

MSF, metacarpal shaft fractures; NHS, National Health Service.

\section{Treatment groups}

Participants will enter a group according to their primary mode of treatment on enrolment into the study. Patients will undergo standard care as per their treating clinician. This will be decided by their clinical care team and will not be affected by their involvement in the study. Treatment groups will be defined as follows;

Surgery: any surgical treatment, defined as insertion of metal via an open or percutaneous approach in an operating theatre, such as open reduction internal fixation, closed reduction internal fixation, intramedullary fixation or wiring, extramedullary wiring or external fixation.

Non-surgical treatment: any 'non-surgical' treatment, defined as regimens with or without reduction (partial or complete) of the fracture, any type of splinting or cast, and/or immediate or delayed mobilisation delivered in a clinic or therapy room environment.

\section{Outcomes}

Participants will be invited to attend a research clinic at 6 weeks following injury. During this visit, clinical assessments of their hand including range of movement, grip strength and presence of rotational deformity or extensor lag will be recorded. Radiographs taken as part of routine clinical care will also be reviewed. The location, fracture morphology, amount of shortening, angulation and presence of step-off deformity on initial radiographs at presentation will be recorded. Complications will be identified by review of the patients' healthcare records at 6 weeks, 3 months and 6 months. Cosmesis will be assessed from question 10 of the Patient Evaluation Measure (PEM), at 6 weeks, 3 months and 6 months. Participant-reported complications will be recorded at the 6-week research clinic visit.

The following PROMs were selected for use following discussions between clinicians, patients, therapists and researchers at the MSF consensus workshop. ${ }^{16}$ They were prioritised due to their ease of use and validity and will be collected at baseline, 6 weeks, 3 months and 6 months.

\section{Patient Evaluation Measure}

The PEM consists of 11 items relating to hand function and appearance, each scored from one to seven from best/normal to worst. It is the PROM of choice in the British Society for Surgery of the Hand (BSSH) National UK Hand Registry (www.ukhr.net) and has been demonstrated to be reliable, valid and responsive for assessing hand disorders. ${ }^{19} 20$ 


$\begin{array}{ll}\text { Table } 2 \text { Eligibility criteria } \\ \text { Inclusion } & \text { Adults } 16 \text { years or older } \\ \text { criteria } & \text { Radiologically confirmed metacarpal shaft } \\ & \text { fracture } \\ & \text { Acute metacarpal shaft fracture affecting } \\ & \text { the index to little finger(s), presenting within } \\ & \text { Willing and able to give informed consent } \\ \text { Exclusion } & \text { Ability to understand English } \\ \text { criteria } & \text { Fracture(s) of the thumb } \\ & \text { Fractures extending into the joint surface } \\ & \text { Fracture(s) of the metaphyseal base and/or } \\ & \text { Fracture(s) associated with dislocation at } \\ & \text { the carpometacarpal joint or other adjacent } \\ & \text { joint dislocation } \\ & \text { Open fractures } \\ & \text { Undisplaced fractures, defined as those } \\ & \text { with a visible fracture line on radiographs } \\ & \text { but anatomical alignment, that is, the bone } \\ & \text { fragments remain aligned with no evidence } \\ & \text { of movement of the fracture fragments } \\ & \text { on anteroposterior, lateral or oblique } \\ & \text { radiographs } \\ & \text { Patients who would not be able to adhere } \\ & \text { questionnaires } \\ & \end{array}$

Patient-reported outcomes measurement information system upper extremity

The PROMIS UE (PROMIS UE Item Bank V.2.0) is a computerised adaptive test, developed by the United States National Institute of Health using item response theory. It has been validated in upper limb fracture and is designed to minimise patient burden and theorised to measure latent traits more precisely than existing PROMs. ${ }^{21}$ For participants who do not engage with electronic means, a paper-based short-form alternative version is available.

\section{Shortened Disabilities of the Arm, Shoulder and Hand Outcome Measure (QuickDASH)}

The Disabilities of the Arm, Shoulder and Hand Score (DASH) is the most commonly used PROM in hand and wrist trauma and has consistently demonstrated good reliability, validity and responsiveness in several psychometric studies. ${ }^{22}$ It consists of 11 items, developed from the original 30-item DASH to improve practicality and eliminate item redundancy. ${ }^{23}$

\section{European Quality of Life Questionnaire (EQ-5D-5L)}

The EQ-5D-5L is a validated, generalised and standardised instrument comprising a Visual Analogue Scale (VAS) measuring self-rated health and a health status instrument, consisting of a five-level response for five domains related to daily activities. ${ }^{24}$ This standardised measure of health status provides a simple, generic measure of health for clinical and economic appraisal. ${ }^{25}$

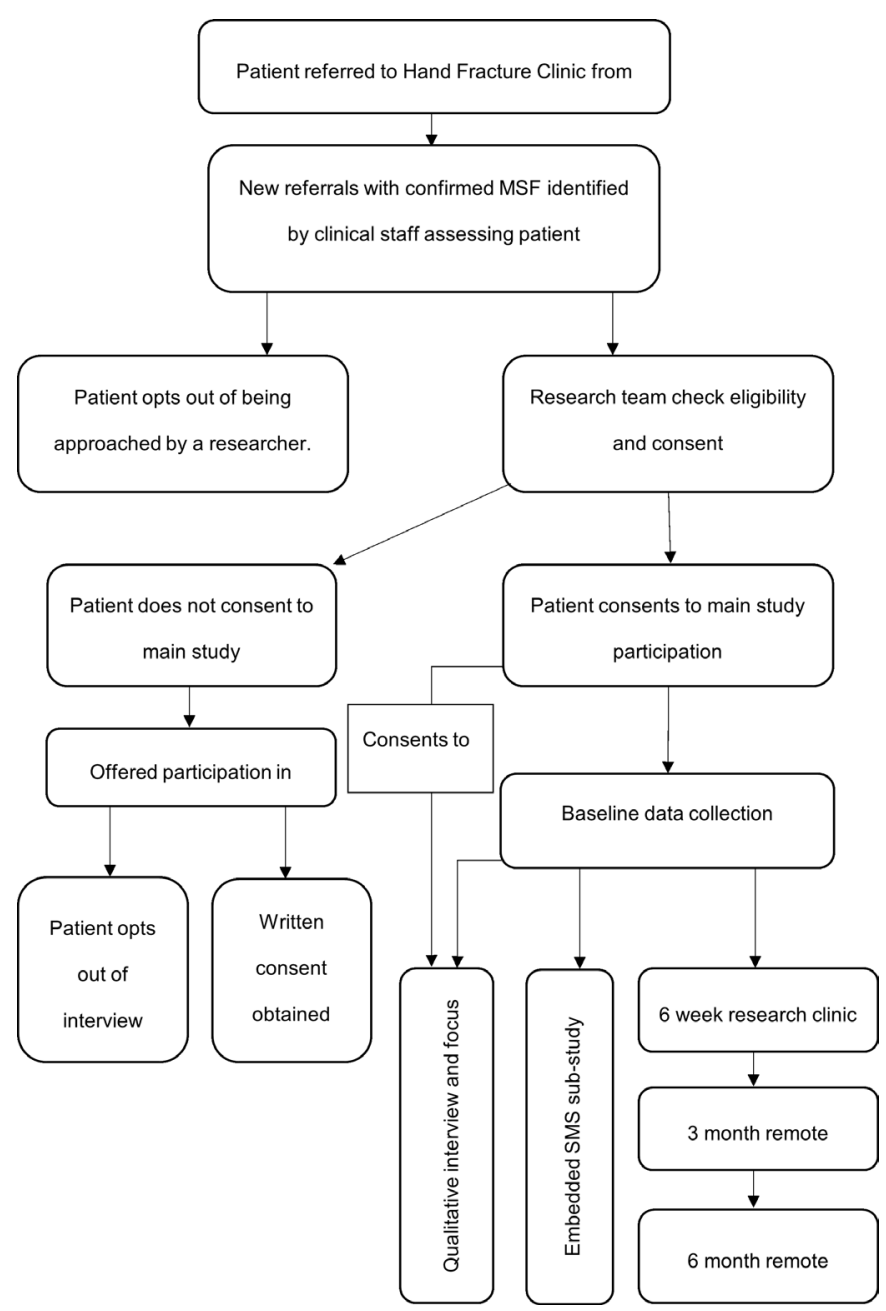

Figure 1 Study flow diagram. MSF, metacarpal shaft fractures. SMS, Short Message Service.

\section{Global Rating of change (GROC)}

The GROC scale is commonly used as an anchor when calculating MCID. It is designed to quantify a patient's improvement or deterioration over time, thus providing a means of measuring self-perceived change in health status. ${ }^{26}$ A 7 -point scale ranging from -3 (very much worse) to +3 (very much better), with 0 indicating 'unchanged', will be used.

\section{Sample size and Justification}

As this is a feasibility study, formal sample size calculations for between-group comparisons are not appropriate. However, we will seek to include as many as 84 participants in the study, aiming for comparable numbers in each treatment group. This sample size will enable estimation of recruitment fraction with margin of error (half width of $95 \% \mathrm{CI}$ ) of $<9$ percentage points and of proportions estimated from the recruited sample, such as completeness of follow-up, to within 13 percentage points.

A purposive sample of 12-16 participants, who indicate a willingness to be interviewed and/or attend focus groups, will be recruited to the qualitative study. Participants who decline to participate in the main cohort study will also be invited for interview. 


\section{Analysis of outcomes}

The outcomes of interest include feasibility outcomes relating to; assessment of eligibility, recruitment and retention rates; completion of follow-up; evaluation of outcome measures and calculation of the MCID for selected outcome measures using quantitative and qualitative assessments; and establishing the feasibility of data collection methods and appropriate time-points for use in a future trial. To address these feasibility aims, data analyses will primarily be descriptive with $95 \%$ CIs to quantify uncertainty in estimates where appropriate.

\section{MCID evaluation}

Minimum clinically important differences for each PROM at 3 months will be estimated using three anchorbased responsiveness statistics: (1) standardised response mean; (2) effect size and (3) Guyatt's Responsiveness Index. An estimation of the MCID will also be calculated by distribution-based methods, using the SE of measurement, SD and effect size. The minimal detectable change will also be calculated to ensure the MCID is greater than the measurement error of the PROM. The sensitivity and specificity of the PROM will be assessed in conjunction with the MCID, as calculated above.

\section{Costing of interventions}

Direct observation of procedures will be used to produce a 'micro-cost' estimate for surgical and non-surgical treatments by combining resource use with unit costs provided by hospital finance departments. Duration of each procedure, theatre staffing, consumables, imaging, supplementary devices, postoperative recovery time and rehabilitation inputs will be recorded from primary sources, such as theatre log systems and patients' electronic and paper clinical records. Standard unit costs will be used to estimate NHS costs of care in the 6-month posttreatment. Unit cost data will be obtained from national databases such as the BNF and PSSRU Costs of Health and Social Care 2019. ${ }^{18}$

There is a need to define the economic morbidity of MSF in terms of the costs of treatment and associated resource use, productivity losses and additional costs incurred by patients during the course of their treatment and recovery. Identifying the costs of treatments will help to establish the feasibility of collecting utility and cost data in a future trial, as well as devising methods for accurately collecting such data to ensure robust cost-effectiveness comparisons in a future trial.

\section{Qualitative analysis}

A thematic, inductive approach as described by Braun and Clarke ${ }^{27}$ will be used to analyse interview and focus group data. We will adopt a six-phase systematic approach consisting of (1) data familiarisation-reading and re-reading the data; (2) generation of initial codes-generating succinct labels (codes) that identify important features of the data that might be relevant to answering the research question; (3) identification of themes through merging and grouping of codes-to identify significant broader patterns of meaning (potential themes) and collating data relevant to each theme; (4) review of generated themeschecking themes against the dataset and refining them where necessary; (5) defining and naming themes-developing a detailed analysis of each and deciding the informative name for each theme and (6) finalisation of themes and generation of a final report-weaving together the analytic narrative and data extracts, and contextualising the analysis in relation to existing literature. NVivo 12 or above Pro software (QSR International Pty, Victoria, Australia) will be used for analysis.

\section{Patient and public involvement statement}

Patients and the public have played a central role in selecting the management of MSF as a key research priority and developing the proposed study. The Hand Surgery Research Prioritisation workshop, attended by clinicians, therapists and patients, recommended the management of MSF as a key research priority. ${ }^{28}$ This was ratified by the James Lind Alliance Priority Setting Partnership on Common Conditions Affecting the Hand and Wrist. ${ }^{16}$ This joint collaboration with the BSSH involved 261 individuals, of which $41 \%$ were patients/carers and $59 \%$ were clinicians. ${ }^{16}$

Furthermore, a MSF Consensus Workshop was held in Nottingham in 2018, bringing together patients with MSF, clinicians, therapists and researchers to share their experiences and develop the PICO framework for a future multicentre trial. ${ }^{16}$ Group discussions informed the eligibility criteria and identified areas of focus for the feasibility work. Selection of patient-centred outcome measures, timing of assessments and follow-up for the proposed study were discussed. A variety of outcome measures were reviewed and the QuickDASH and PROMIS-UE were subsequently added. One clinic visit in addition to virtual follow-up was included and follow-up was adjusted to 3 and 6 months following patient discussions. Feedback from patients who attended the workshop has informed all aspects of the research including research design, choice of outcome measures and length and location of follow-up.

The study protocol, participant information sheets and consent forms have been reviewed by PPI members, with feedback provided to optimise their utility. We have set up a Metacarpal Shaft Fracture Patient Advisory Group to inform the design, delivery and output of the research. An electronic PPI platform has been created on the Centre for Evidence Based Hand Surgery (CEBHS) website to encourage regular input from patients/public.

Feedback from the Metacarpal Shaft Fracture Patient Advisory Group will guide distribution of findings to the public. This will include, but not be limited to, newsletters, local media outlets, events and plain English summaries of all published journal articles. 
Incorporating patient and public involvement in all aspects of the research pathway, from research design to co-development and review of all study documents, supports our commitment to ensuring sustained and meaningful PPI throughout the study.

\section{Study management}

The study is funded by a National Institute for Health Research Doctoral Fellowship awarded to Miss Rowa Taha (NIHR300197). It is sponsored by the University of Nottingham and will be managed and co-ordinated from the Centre for Evidence Based Hand Surgery (CEBHS), University of Nottingham.

\section{ETHICS AND DISSEMINATION}

\section{Ethics committee and regulatory approvals}

This study has received approval from the Research Ethics Committee (REC), the respective NHS Research \& Development (R\&D) departments and the Health Research Authority (HRA) (REC reference 20/EE/0124). It will be conducted in accordance with the ethical principles that have their origin in the Declaration of Helsinki ${ }^{29}$; the principles of Good Clinical Practice ${ }^{30}$ and the UK Department of Health Policy Framework for Health and Social Care, $2017 .^{31}$

\section{Informed consent}

The process for obtaining participant informed consent will be in accordance with the REC guidance and Good Clinical Practice. ${ }^{30}$ The investigator or their nominee and the participant or other legally authorised representative shall both sign and date the informed consent form before the person can participate in the study. Written informed consent will be separately sought for taking part in the qualitative study.

\section{Safety considerations and data protection}

There are no significant safety issues with this study. The study is observational and does not interfere with the routine clinical care pathway. The treatments participants receive are widely available within the National Health Service and will not be altered by taking part in the study. The questionnaires, interviews and focus groups are neither burdensome nor contain sensitive questions and all reasonable expenses, such as travel and parking costs, associated with attending research visits will be fully reimbursed.

All study staff and investigators will endeavour to protect the rights of the study's participants to privacy and informed consent and will adhere to the Data Protection Act, 2018. ${ }^{32}$ Case report forms will be held securely and access to the information will be limited to the study staff, investigators and relevant regulatory authorities. Computer held data including the study database will be held securely and password protected. All data will be stored on a secure dedicated web server. Access will be restricted by user identifiers and passwords (encrypted using a one-way encryption method).

\section{Dissemination}

Results of this study will be reported fully and made publicly available when the research has been completed. The outcomes of the study will be published in suitable peer-reviewed journals and will be reported locally, nationally and internationally in the form of presentations. Reporting will be in compliance with Strengthening the Reporting of Observational Studies in Epidemiology (STROBE) guidelines. $^{33}$ In order to fulfil reporting guidelines, a copy of the research paper will also be sent to the National Institute for Health Research (NIHR) programme issuing the funding contract.

The findings will be presented at national and international meetings of relevant scientific societies. We will also publish key findings on the CEBHS website, and via the 'Hand Evidence Updates', distributed by the CEBHS to over 800 national and international members.

Social media platforms will also be used to maximise dissemination of key findings as supported by evidence from the Nottingham Clinical Trials Unit. ${ }^{34} 35$

\section{Twitter Rowa Taha @FACTSstudy1}

Contributors The study concept and design was conceived by RT, PL, AM, TD and AK. PL advised on qualitative study design, data collection and analysis considerations. CB advised on study design, clinical assessments and data collection. RT prepared the first draft of the manuscript. All authors provided edits and critiqued the manuscript for intellectual content.

Funding This work was supported by the National Institute for Health (NIHR) research grant number NIHR300197 and the British Society for Surgery of the Hand (BSSH).

Disclaimer The views expressed in this publication are those of the author(s) and not necessarily those of the NHS, the National Institute for Health Research or the Department of Health and Social Care.

Competing interests RT, AK and AM report grants from the National Institute for Health Research (NIHR) and the British Society for Surgery of the Hand (BSSH). This protocol is independent research supported by the National Institute for Health Research (NIHR Doctoral Fellowship - Stage 2, Miss Rowa Taha, NIHR300197).

Patient and public involvement Patients and/or the public were involved in the design, or conduct, or reporting, or dissemination plans of this research. Refer to the Methods section for further details.

Patient consent for publication Not required.

Provenance and peer review Not commissioned; externally peer reviewed.

Open access This is an open access article distributed in accordance with the Creative Commons Attribution 4.0 Unported (CC BY 4.0) license, which permits others to copy, redistribute, remix, transform and build upon this work for any purpose, provided the original work is properly cited, a link to the licence is given, and indication of whether changes were made. See: https://creativecommons.org/ licenses/by/4.0/.

ORCID iD

Rowa Taha http://orcid.org/0000-0002-2403-5107

\section{REFERENCES}

1 van Onselen EBH, Karim RB, Hage JJ, et al. Prevalence and distribution of hand fractures. J Hand Surg Br 2003;28:491-5.

2 Gudmundsen TE, Borgen L. Fractures of the fifth metacarpal. Acta Radiol 2009;50:296-300.

3 Stanton JS, Dias JJ, Burke FD. Fractures of the tubular bones of the hand. J Hand Surg Eur Vol 2007;32:626-36. 
4 Hove LM. Fractures of the hand. distribution and relative incidence. Scand J Plast Reconstr Surg Hand Surg 1993;27:317-9.

5 Laugharne E, Bhavsar D, Rajaratnam V. The distribution of hand fractures: a British perspective. Eur J Plast Surg 2013;36:367-70.

6 Accident and Emergency Statistics. Demand, performance and pressure: house of commons library, 2017.

7 Angermann P, Lohmann M. Injuries to the hand and wrist. A study of 50,272 injuries. J Hand Surg Br 1993;18:642-4.

8 de Jonge JJ, Kingma J, van der Lei B, et al. Fractures of the metacarpals. A retrospective analysis of incidence and aetiology and a review of the English-language literature. Injury 1994;25:365-9.

9 Nakashian MN, Pointer L, Owens BD, et al. Incidence of metacarpal fractures in the US population. Hand 2012;7:426-30.

10 Wong JYP. Time off work in hand injury patients. J Hand Surg Am 2008;33:718-25.

11 Giddins GEB. The non-operative management of hand fractures. $J$ Hand Surg Eur Vol 2015;40:33-41.

12 Diaz-Garcia R, Waljee JF. Current management of metacarpal fractures. Hand Clin 2013;29:507-18.

13 Kollitz KM, Hammert WC, Vedder NB, et al. Metacarpal fractures: treatment and complications. Hand 2014;9:16-23.

14 Taha RHM, Grindlay D, Deshmukh S, et al. A systematic review of treatment interventions for metacarpal shaft fractures in adults. Hand 2020:1558944720974363.

15 Westbrook AP, Davis TRC, Armstrong D, et al. The clinical significance of Malunion of fractures of the neck and shaft of the little finger metacarpal. J Hand Surg Eur Vol 2008;33:732-9.

16 Centre for Evidence Based Hand Surgery, University of Nottingham. Hand fracture research workshop: surgical versus non-surgical treatment of Metacarpal Shaft Fractures [Internet], 2018. Available: https://www.nottingham.ac.uk/research/groups/cebhs/documents/ research-documents/2018-workshop-programme.pdf

17 Macdonald BB, Higgins A, Kean S, et al. Long-term follow-up of unoperated, nonscissoring spiral metacarpal fractures. Plast Surg 2014;22:254-8.

18 Curtis L, Burns A. Unit costs of health and social care 2019, personal social services research unit. Canterbury: University of Kent, 2019.

19 Dias JJ, Bhowal B, Wildin CJ, et al. Assessing the outcome of disorders of the hand. is the patient evaluation measure reliable, valid, responsive and without bias? J Bone Joint Surg Br 2001;83:235-40.

20 Dias JJ, Rajan RA, Thompson JR. Which questionnaire is best? the reliability, validity and ease of use of the patient evaluation measure, the disabilities of the arm, shoulder and hand and the Michigan hand outcome measure. J Hand Surg Eur Vol 2008;33:9-17.

21 Gausden EB, Levack AE, Sin DN, et al. Validating the patient reported outcomes measurement information system (PROMIS) computerized adaptive tests for upper extremity fracture care. J Shoulder Elbow Surg 2018;27:1191-7.

22 Dacombe PJ, Amirfeyz R, Davis T. Patient-Reported outcome measures for hand and wrist trauma: is there sufficient evidence of reliability, validity, and responsiveness? Hand 2016;11:11-21.

23 Beaton DE, Wright JG, Katz JN, et al. Development of the QuickDASH: comparison of three item-reduction approaches. J Bone Joint Surg Am 2005;87:1038-46.

24 Brooks R. EuroQol: the current state of play. Health Policy 1996;37:53-72.

25 EuroQol Group. EuroQol--a new facility for the measurement of health-related quality of life. Health Policy 1990;16:199-208.

26 Kamper SJ, Maher CG, Mackay G. Global rating of change scales: a review of strengths and weaknesses and considerations for design. J Man Manip Ther 2009;17:163-70.

27 Braun V, Clarke V. Using thematic analysis in psychology. Qual Res Psychol 2006;3:77-101.

28 Centre for Evidence Based Hand Surgery, University of Nottingham. CEBHS Hand Fracture Research Workshop [Internet], 2016. Available: https://www.nottingham.ac.uk/research/groups/cebhs/ documents/research-documents/cebhs-hand-fracture-workshopreport.pdf [Accessed 23 Nov 2018]

29 World Medical Association. World Medical association Declaration of Helsinki: ethical principles for medical research involving human subjects. JAMA 2013;310:2191-4.

30 International Conference on Harmonisation of technical requirements for registration of pharmaceuticals for human use. ICH harmonized tripartite guideline: Guideline for Good Clinical Practice [Internet]. Available: https://database.ich.org/sites/default/files/E6_R2 Addendum.pdf [Accessed 23 Nov 2018].

31 NHS Health Research Authority. UK policy framework for health and social care research. v3.3 edn, 2017: 1-35. https://www.hra.nhs.uk/ media/documents/Final_Accessibility_uk-policy-framework-healthsocial-care-research.pdf

32 Data Protection Act 2018, c. 12. Available: http://www.legislation. gov.uk/ukpga/2018/12/contents/enacted

33 von Elm E, Altman DG, Egger M, et al. The strengthening the reporting of observational studies in epidemiology (STROBE) statement: guidelines for reporting observational studies. Lancet 2007;370:1453-7.

34 Adams CE, Jayaram M, Bodart AYM, et al. Tweeting links to Cochrane schizophrenia group reviews: a randomised controlled trial. BMJ Open 2016;6:e010509.

35 Buckarma EH, Thiels CA, Gas BL, et al. Influence of social media on the dissemination of a traditional surgical research article. J Surg Educ 2017;74:79-83. 